\title{
Comportamiento mecánico de un sistema geopolimérico sintetizado a partir de escoria blanca
}

\section{Mechanical behavior of a synthetic polymeric system from white scan}

\author{
Juan Esteban Ochoa-Franco*, Alfonso López-Díaz ${ }^{\mathbb{D}}$, Oscar Javier \\ Gutiérrez-Junco ${ }^{\mathbb{D}}$ y Daniel Fernando Ochoa-Franco \\ Universidad Pedagógica y Tecnológica de Colombia, Colombia
}

\begin{abstract}
Resumen. En este artículo se evalúa la formación de un geopolímero a partir de escoria de horno cuchara como precursor. Como activador se utilizó hidróxido de sodio 12 Molar y silicato de sodio. Se evaluaron las propiedades mecánicas de compresión y absorción de agua para la formación de un geopolímero, con adiciones de residuos cerámicos (RC). Se prepararon 32 muestras de pasta distribuidas en 8 mezclas con diferentes dosificaciones. La escoria y el RC tenían un tamaño de partícula de $150 \mu \mathrm{m}$. La variación en peso del silicato de sodio fue de $5 \%$ $10 \%, 15 \%$ y $20 \%$. Se añadió RC en proporciones de peso de $5 \%, 10 \%, 15 \%$ y $20 \%$. El curado de la pasta se hizo a temperatura ambiente durante 24 horas. Los resultados obtenidos indican que el tipo de geopolímero formado durante la reacción, adquiere propiedades mecánicas adecuadas para ser utilizado en la industria de la construcción en la elaboración de mampuestos, dándole un aprovechamiento industrial a la escoria y con beneficio ambiental.
\end{abstract}

Palabras Claves. Geopolímeros; escoria blanca; hidróxido de sodio.

Resumen. In this article, the formation of a geopolymer as a precursor is evaluated. 12 Molar sodium hydroxide and sodium silicate are applied as activator. The mechanical properties of compression and water absorption for the formation of a geopolymer were evaluated, with additions of ceramic waste (RC). 32 paste samples distributed in 8 mixtures with different dosages were prepared. The slag and the RC have a particle size of $150 \mu \mathrm{m}$. The weight variation of sodium silicate was $5 \% 10 \%, 15 \%$ and $20 \%$. RC was added in weight proportions of $5 \%$, $10 \%, 15 \%$ and $20 \%$. The curing of the paste was done at room temperature for 24 hours. The results indicate that the type of geopolymer forms during the reaction, acquires encouraging mechanical properties for use in the construction industry in the preparation of masonry, gives an industrial use to the slag and with environmental benefit.

Keywords. Geopolymers; white slag; sodium hidroxides.

Como Citar. J.E. Ochoa-Franco, A. López-Díaz, O.J. Gutierrez-Junco y D.F. Ochoa-Franco, "Comportamiento mecánico de un sistema geopolimérico sintetizado a partir de escoria blanca", Jou. Cie. Ing., vol. 12, no. 1, pp. 253-263, 2020. doi:10.46571/JCI.2020.1.22

Recibido: 17/04/2020 Revisado: 06/07/2020 Aceptado: 21/08/2020 


\section{Introducción}

Los residuos sólidos generados durante los procesos siderúrgicos de extracción y refinación de los metales se llaman escoria. Estos materiales se componen principalmente de óxidos y silicatos de magnesio, calcio, aluminio, hierro. La escoria alcanza cantidades de hasta $15 \%$ por tonelada de carga en cada colada, dependiendo de la homogeneidad del material. Otros factores que pueden modificar la composición de la escoria son la cantidad de ferroaleaciones que se añadan, el tipo de horno que se utilice, entre otras.

Actualmente la producción anual mundial de ladrillos es de unos 1,391'000,000,000 unidades y la demanda de ladrillos está continuamente en aumento [1]. Los ladrillos convencionales se producen a partir de arcilla, en un horno de cocción de alta temperatura o de cemento para construcción. Los hornos no sólo consumen cantidad significativa de energía, sino que producen gran cantidad de gases con efecto invernadero. Un ladrillo de arcilla, en promedio, incorpora una energía de $2,0 \mathrm{Kw} / \mathrm{h}$ y libera unos $0,41 \mathrm{~kg}$ de dióxido de carbono $\left(\mathrm{CO}_{2}\right)$ [2]. Así mismo, se afirma que para el proceso de manufactura de una tonelada de cemento portland ordinario se libera alrededor de una tonelada de $\left(\mathrm{CO}_{2}\right)$ a la atmósfera [3].

Se hace necesario dar uso a la escoria y residuos sólidos de la producción de acero, en un producto de bajo costo que cumpla con estándares de calidad internacionales. Desde este punto de vista, los geopolímeros son materiales que ofrecen una opción económicamente rentable y amigable con el medio ambiente. Comparables con materiales puzolánicos que comúnmente se usan en la producción de mampostería de cemento Portland. Estos materiales por sí solos carecen de propiedades cementantes pero que en condiciones apropiadas aportan endurecimiento y durabilidad al material [4]

Siguen siendo insuficientes las investigaciones de producción de ladrillos mediante geopolimerización, en el que se dé un aprovechamiento a residuos industriales. Muchos investigadores sustituyeron la arcilla común por ceniza volante tipo $\mathrm{F}$, encontrando mayor resistencia a la compresión comparada con un ladrillo de arcilla convencional [5]. Estas investigaciones pretenden incorporar iones activadores de sodio, potasio y litio entre otros, en condiciones de curado a temperaturas superiores a $\operatorname{los} 40^{\circ} \mathrm{C}$ y humedad relativa entre $30 \%$ y $100 \%[6,10]$. Todas las investigaciones anteriores obtuvieron mejoras en la resistencia mecánica del material sometido a esfuerzo. Otros investigadores sustituyeron el cemento portland por escoria de alto horno, mejorando la propiedades cuando la escoria no excedía el $20 \%$, además de disminuir el tiempo de curado a 7 días [11].

Las escorias presentan diferentes composiciones químicas, al igual que la fase vítrea. Los contenidos de $\mathrm{SiO}_{2}, \mathrm{CaO}$ y $\mathrm{Al}_{2} \mathrm{O}_{3}$ son variables y reaccionan con estequiometría diferente en los procesos de hidratación, carbonatación y reducción. La velocidad de hidratación dependerá de la reactividad y cantidad de hidróxido de calcio presente en las mezclas hidratadas [12]. Los geopolímeros se obtienen a partir de mezclas en óptimas proporciones de un mineral o compuesto basado en $\mathrm{Al}_{2} \mathrm{O}_{3}$ y $\mathrm{SiO}_{2}$ (precursores) con un agente químico (activador alcalino), el cual promueve una serie de reacciones que conducen a la formación de un producto con características cementantes. Los activadores son por lo general soluciones altamente alcalinas, constituidas por $\mathrm{NaOH}$ o $\mathrm{KOH}$ donde el tipo de ión alcalino tiene efecto sobre las características y propiedades mecánicas finales del producto [13]. Bajo este principió se ha demostrado que desechos industriales, pueden ser considerados precursores en la formación de geopolímeros con resistencias a la compresión elevadas, si se activan en un medio altamente alcalino con $\mathrm{NaOH}$ y $K O H[14,17]$.

El presente trabajo evaluó las propiedades mecánicas del uso de escoria blanca como precursor, activada con Hidróxido de Sodio y Silicato de Sodio. De acuerdo a los compuestos químicos formados por las reacciones entre la escoria y los activadores, se evidenciaron procesos de formación geopolimérica. Para tales efectos se confeccionaron pastas con diferentes porcentajes 
de silicato de sodio con adiciones de RC. Los resultados experimentales fueron utilizados para describir la influencia del hidróxido de sodio y el silicato de potasio en el comportamiento mecánico del material geopolimérico elaborado.

\section{Parte Experimental}

La parte experimental incluyó la manufactura de mezclas constituidas de Escoria Blanca, con diferentes proporciones en peso de HidrÓxido de Sodio (Soda cáustica en escama), Silicato de Sodio Reactivos RD ", con una densidad de 1,35 y 122 P.M y RC. Los ensayos se realizaron sobre 32 muestras cilíndricas de $25 \mathrm{~mm}$ de diámetro x $50 \mathrm{~mm}$ de altura.

\subsection{Materiales}

En este estudio se utilizó Escoria Blanca provenientes de la empresa Sidenal S.A (Colombia).

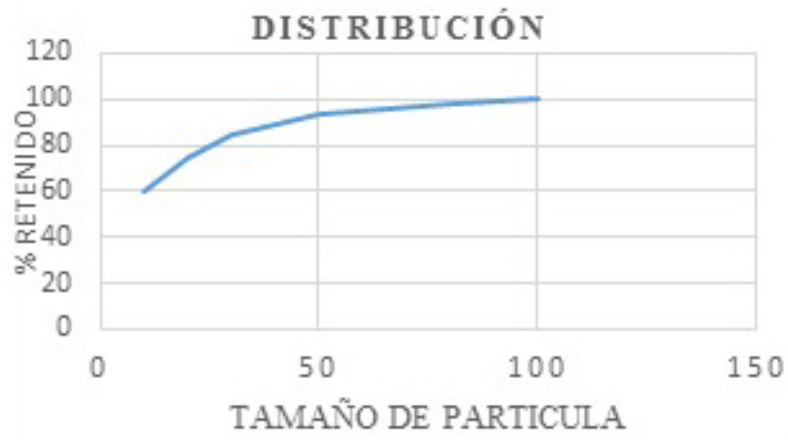

Figura 1: Distribución granulométrica escoria blanca del horno cuchara

En la figura 1 se muestra su distribución granulométrica, el RC proviene de desechos de teja de arcilla tomados de desechos construcción de la Universidad Pedagógica y Tecnológica de Colombia. La caracterización de estos materiales se realizó mediante Fluorescencia de Rayos X, su composición química se muestra en la tabla 1.

\begin{tabular}{|c|c|c|}
\hline Compuesto & Escoria Blanca $\%$ & RC \\
\hline $\mathrm{SiO}_{2}$ & 23,51 & 52,91 \\
\hline $\mathrm{Al}_{2} \mathrm{O}_{3}$ & 6,37 & 25,70 \\
\hline $\mathrm{F}_{2} \mathrm{O}_{3}$ & 3,80 & 6,93 \\
\hline $\mathrm{Mn}_{2} \mathrm{O}_{3}$ & 3,63 & \\
\hline $\mathrm{CaO}$ & 46,1 & 0,543 \\
\hline $\mathrm{Mg} \mathrm{g}_{2} \mathrm{O}$ & 13,28 & 0,543 \\
\hline $\mathrm{K}_{2} \mathrm{O}$ & $<0,01$ & 1,428 \\
\hline $\mathrm{P}_{2} \mathrm{O}_{5}$ & $<0,01$ & 0,021 \\
\hline $\mathrm{Na}_{2} \mathrm{O}$ & 0,05 & $<0,01$ \\
\hline $\mathrm{SO}_{3}$ & 0,39 & 1,129 \\
\hline
\end{tabular}

Tabla 1: Fluorescencia de rayos X, material prima empleada. Fuente: Autores.

Como agregado fino se utilizó el residuo cerámico con un tamaño de grano $150 \mu m$ y absorción del $7 \%$. 
La Escoria Blanca enfriada del horno cuchara apilada en la empresa se muestreo aleatoriamente y se trasladó al laboratorio donde se caracterizó por Fluorescencia de Rayos X y Difracción de Rayos X, según se muestra en la Figura 2 y la Tabla 1.

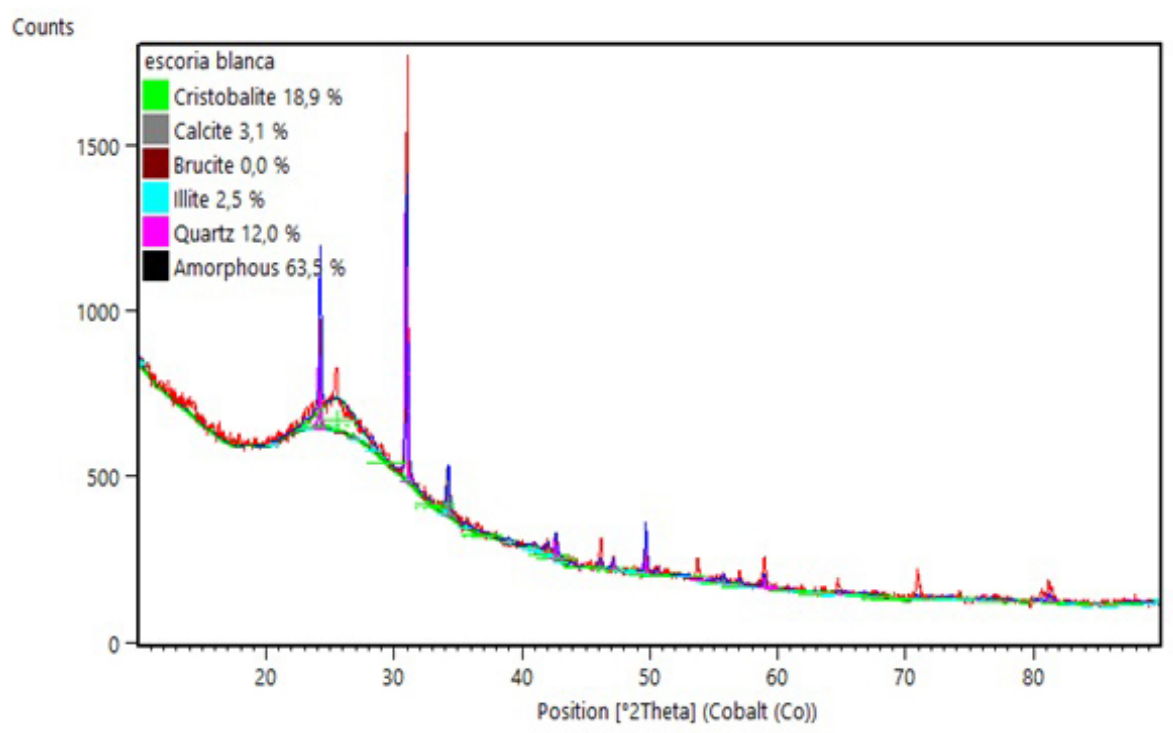

Figura 2: Difracción de la escoria blanca.

A continuación se obtuvo un tamaño de partícula de $150 \mu m$, en un tamiz de malla número 100 con ayuda de un molino de bolas y una pulverizadora. De acuerdo a la literatura el hidróxido de sodio 12 Molar y el silicato de sodio 5 Molar, propisia las mejores condiciones de reacción para alcanzar mayores durezas.

\subsection{Preparación de las muestras}

Se prepararon 3 mezclas de escoria Blanca con adiciones parciales de Silicato de Sodio y RC, cada muestra tuvo dos replicas.

Las abreviaturas EB, CH, HS y SS se utilizaron para identificar las muestras Escoria Blanca, Hidróxido de Sodio y Silicato de Sodio, respectivamente por sus iniciales. También fueron introducidos a la nomenclatura los porcentajes de sustitución del material cementante principal: por ejemplo, EB7-HS3 significa que la mezcla es de $70 \%$ Escoria Blanca y $30 \%$ Hidróxido de Sodio; EB7-CH2-SS1 representa la mezcla de 70\% Escoria Blanca, 20\% RC y $10 \%$ Silicato de Sodio; EB5-HS25-SS15 representa la mezcla de $60 \%$ Escoria Blanca, $25 \%$ Hidróxido de Sodio y $15 \%$ Silicato de Sodio.

\section{Ensayos y descripción de equipos}

\subsection{Resistencia a la compresión}

Todas la muestras fueron sometidas a ensayos mecánicos de comprensión en la Maquina de Esfuerzos universal. Con los resultados obtenidos se analizaron las propiedades mecánicas de la adición de Silicato de Sodio, para lo cual se elaboraron la muestras de $25 \mathrm{~mm}$ de diámetro x $50 \mathrm{~mm}$ de alto (ver Figura 3), a edades de 7 y 28 días, siguiendo la recomendación de relación de esbeltez 1:2 dada en la norma ASTM C39 (2015) [18]. 


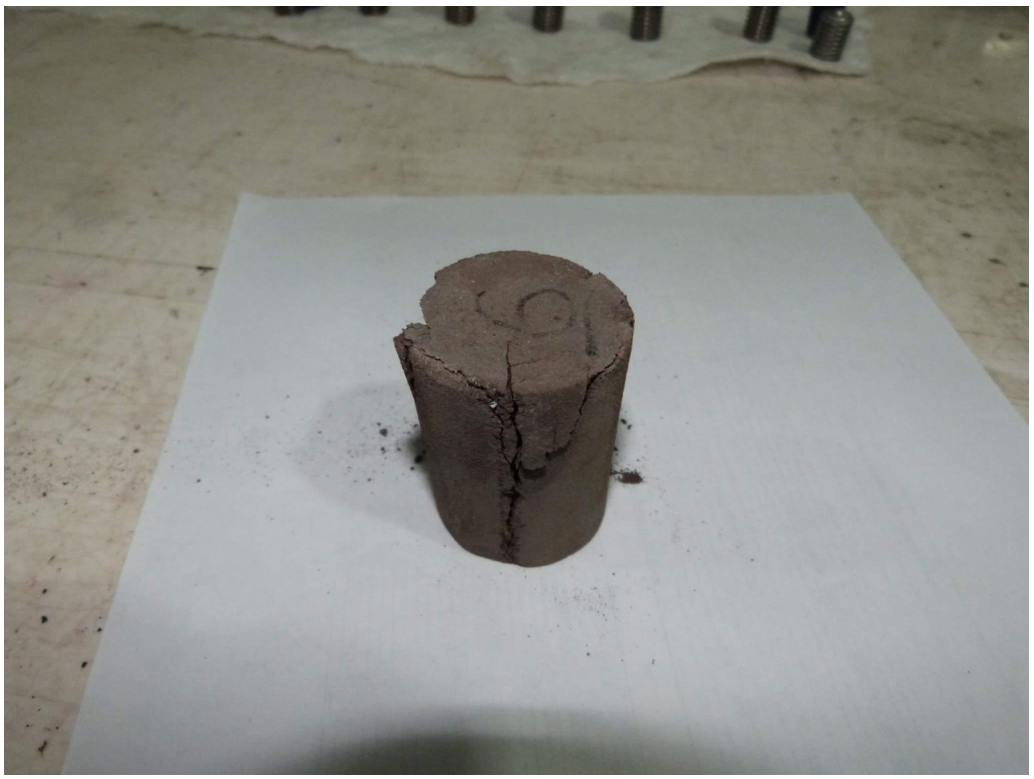

Figura 3: Muestra EN80-SS20.

\subsection{Absorción de agua}

Con el fin de determinar el comportamiento del nuevo material frente a la absorción de agua, se evaluó esta propiedad en base a la norma ASTM 1585 (2013) [19]. Para este ensayo las probetas se sumergen en agua por 24 horas para después comparar diferencias de peso.

\subsection{Difracción de rayos x y microscopía electrónica de barrido}

Las muestras fueron caracterizadas mediante un difractometro de rayos X (DRX) del ICITEMA (Instituto para la Investigación y la Innovación). El ajuste experimental usado es el

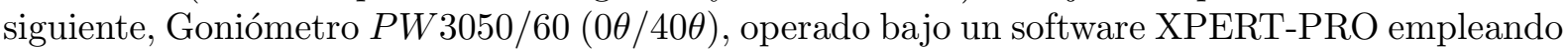
una radiación monocromática de $C o K \alpha 1,54 \AA$, operado a $40 \mathrm{kV}$ y $40 \mathrm{~mA}$ bajo condiciones de temperatura ambiente. Por otro lado, se emplearon parámetros estadísticos tales como el coeficiente de variación (CV) y la media aritmética (X). Adicionalmente se empleo el coeficiente de correlación (r) el cual permitió medir el grado de intensidad de relación entre los valores calculados y los medidos.

\section{Resultados y discusión}

\subsection{Resistencia a la compresión}

Los resultados de los ensayos de resistencia a compresión, fc, para las muestras a edades de 7 y 28 días se muestran en la tabla 2. De acuerdo a la anterior tabla se puede concluir que las muestras aumentan la resistencia mecánica gracias a mayores contenido de $\mathrm{SiO}_{2}$ y $\mathrm{Al}_{2} \mathrm{O}_{3}$, minerales que son responsables de la formación de las nuevas cadenas geopoliméricas. Su composición química se asemeja a la ceniza volante, material que dio inicio a la geopolimerización [20-22]. Debido a que los silicatos y aluminosilicatos ceden y ganan electrones con los hidróxidos $O H-\mathrm{De}$ igual modo la muestra EB70 - CH10 - SS15 - HS5 mostro los mejores comportamientos mecánicos en resistencia a la compresión con 18,49MPa. Las muestras cumplen con la norma ASTM C39 de resistencia a la compresión, de mismo modo las muestras EB75-CH10 - SS20; EB60 - CH20 - SS15; también cumplen con la normas ASTM C39. De acuerdo a este comportamiento mecánico se puede concluir que el uso de escoria blanca procedente de horno cuchara, es un material adecuado para la formación de geopolímeros con resistencia mecánica adecuada para uso industrial 


\subsection{Absorción de agua}

Se realizaron pruebas de absorción a todas las muestras, siguiendo la norma ASTM C 69 de las cuales se determinó la absorción promedio inicial es de $12 \%$ y un promedio de absorción general de 14\%, que según la norma ASTM C 89 es adecuada para el uso en mampostería estructural.

\subsection{Difracción de rayos $X$}

En la Figura 4 se muestra los resultados de Difracción de Rayos X de la muestra EB70 $C H 10-S S 15$ que es representativa y con los mejores resultados, en ella se observo la abundancia de fases cristalográficas en la muestras ternarias hidratadas a causa de los elevados porcentajes de escoria siderúrgica y RC presentes.

\begin{tabular}{|c|c|c|c|c|c|c|c|c|}
\hline \multirow[t]{2}{*}{ Identificación } & \multicolumn{4}{|c|}{$F_{c}, 7$ Días, $M P a$} & \multicolumn{4}{|c|}{$F_{c}, 28$ Días, $M P a$} \\
\hline & Muestra 1 & Muestra 2 & Muestra 3 & $X$ & Muestra 1 & Muestra 2 & Muestra 3 & $X$ \\
\hline EB80-SS15-HS5 & 7,07 & 7,63 & 6,13 & 6,94 & 17,07 & 17,63 & 17,13 & 17,27 \\
\hline EB75-CH15-SS10 & 6,56 & 6,12 & 5,88 & 6,18 & 16,56 & 17,12 & 16,88 & 16,85 \\
\hline EB75-CH10-SS15-HS5 & 5,16 & 5,72 & 6,48 & 5,78 & 16,16 & 17,72 & 16,48 & 16,78 \\
\hline EB70-CH15-SS15 & 9,72 & 8,92 & 6,68 & 8,44 & 16,72 & 18,20 & 17,68 & 17,53 \\
\hline EB70-CH10-SS15-HS5 & 7,78 & 8,56 & 9,66 & 8,66 & 18,70 & 18,56 & 19,66 & 18,97 \\
\hline EB65-CH20-SS15 & 6,99 & 6,19 & 6,49 & 6,55 & 16,99 & 15,39 & 17,49 & 16,62 \\
\hline EB65-CH15-SS15-HS5 & 9,87 & 9,07 & 9,57 & 9,50 & 16,87 & 18,07 & 16,57 & 17,17 \\
\hline EB60-CH25-SS15 & 4,32 & 5,52 & 4,72 & 4,85 & 15,32 & 18,52 & 15,72 & 16,52 \\
\hline EB60-CH20-SS15-HS5 & 3,35 & 4,75 & 3,85 & 3,98 & 14,35 & 15,75 & 16,85 & 15,65 \\
\hline EB55-CH30-SS15 & 3,78 & 3,32 & 3,62 & 3,57 & 13,78 & 13,32 & 12,62 & 13,24 \\
\hline EB55-CH25-SS15-HS5 & 3,87 & 2,77 & 3,28 & 3,28 & 12,87 & 13,77 & 11,21 & 12,58 \\
\hline
\end{tabular}

Tabla 2: Proporciones dé\% en peso de las mezclas.

En las plantas de fundición de acero la escoria es enfriada rápidamente en posos con agua, la velocidad de enfriamiento depende del tamaño de la partícula: las partículas grandes enfrían más lentamente permiten la cristalización en su interior. Por otro lado, algunas de las partículas voluminosas estallan y forman pequeñas gotas fundidas. Otras partículas se enfrian de la misma forma que las partículas sólidas cuando pero cuando abandonan definitivamente la llama y se conservan como esferas huecas con variaciones en el espesor de los bordes. Además, una consecuencia de la presencia de gases $\left(\mathrm{CO}_{2}, \mathrm{O}_{2}, \mathrm{SO}_{2}\right.$ y $\left.\mathrm{H}_{2} \mathrm{O}\right)$ en su posible acción en las reacciones secundarias con el cristal que conllevan a la polimerización o despolimerización de las moléculas de sílice en las partículas de ceniza [23]. En el espectro de difracción de rayos $\mathrm{X}$ mostrado en la figura 5 se observa el material geopolimérico evaluado. Se aprecia que es altamente cristalino y sus formaciones son características de una escoria activada en un medio altamente alcalino. En el espectro se determina que el sistema está compuesto por un gran porcentaje por Periclase, también conocida como óxido de Magnesio $(\mathrm{MgO})$ con un numero de PDF 00-004-0829 (PDF: Powder Difracction File). Estabilizado con un metal alcalino y de sistema cristalino cúbico hexahedral al igual que lo reportado por otros investigadores [24-26]. El $M g O$ aumenta el tamaño de grano de las partículas, además de, aumentar la fragilidad de geopolímero $[27,28]$.

Otro compuesto encontrado es la Magnetita, con formula química $\mathrm{Fe}_{3} \mathrm{O}_{4}$, con un numero de (PDF 04-012-7038), característico de los geopolímeros basados en cenizas volantes [29-31] y geopolímeros a base de escoria [26,32]. Este material Cristaliza en el sistema cúbico con más de $72 \%$ de hierro, responsable de buenas propiedades mecánicas [33]. 


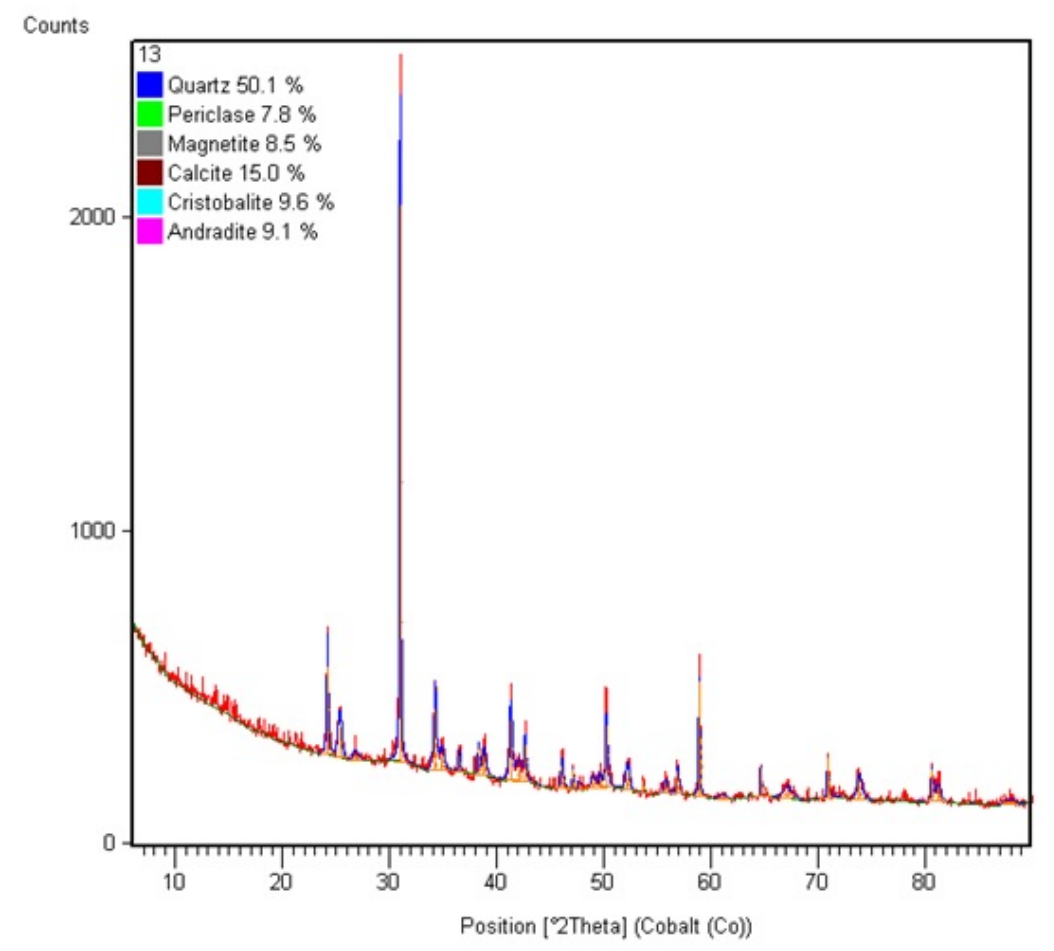

Figura 4: DRX de la muestra de Geopolímero EB70-CH10-SS15-HS5.

La calcita es también observada en el DRX, con formula química $\left(\mathrm{CaCO}_{3}\right)$, y con un numero de (PDF 00-83-0577). La calcita está presente en varias rocas naturales como la piedra caliza. Es un compuesto principalmente de carbonato de calcio asociado a otras fases cristalinas como la dolomita (calcio-magnesio-carbonato) $\mathrm{Ca}, \mathrm{Mg},\left(\mathrm{CO}_{3}\right)_{2}$ y otras impurezas. La calcita ha sido ampliamente estudiada en la influencia de la fragilidad de los geopolímeros y materiales activados alcalinamente [34-36]. Cuando la calcita está presente hasta el 20\%, mejora las propiedades mecánicas de compresión y tensión de geopolímeros [37]. Por encima de este contenido, las propiedades mecánicas disminuyen y la contracción aumenta. Esta pérdida de propiedades se debe al mayor contacto de los iones de calcio y magnesio entre las partículas además de la mayor distribución de las partículas del gel polimérico [38].

Otro compuesto característico de los geopolímeros y también es evidenciado es la andradita $\left(\mathrm{Ca}_{3} \mathrm{Fe}_{2}\left(\mathrm{SiO}_{4}\right)_{3}\right)$ [39,40], con un número (PDF No. 00-89-7563). Es un mineral de gran dureza y propiedades mecánicas excelentes. Se deduce que se logró formar parcialmente a partir de la illita, y otra parte desde el material amorfo. Es posible obtener estructuras más complejas a partir de la andradita y obtener valores más altos de resistencia a la compresión [41].

La Cristobalita con número (PDF 00-039-1425), se encuentra de forma natural en la arcilla, que al ser adicionada en el RC permaneció constante después del proceso de geopolimerización. Para su transformación se haría necesario temperaturas superiores a los $800^{\circ} \mathrm{C}$. Este material ayuda a la formación de gel polimérico [42]. De otra parte el cuarzo $\mathrm{SiO}_{2}$ con número PDF 00-85-0865, presente en la escoria reaccionar parcialmente.

\subsection{Microscopía electrónica de barrido}

A continuación se muestran las imágenes logradas con un microscopio electrónico de barrido marca Zeiss Evo ma 10, previo alistamiento de la muestras en el sistema de recubrimiento de bombeo rotatorio compacto adecuado para Spaterim o SEM. Para lo cual se tomaron dos muestras, que presentaron el mejor comportamiento mecánico EB70-CH10-SS15-HS5. De 
acuerdo, a lo observado en la Figura 5(a), no se apreció micro porosidad importante a lo largo de la microestructura. Esta imagen fue la única detectada a lo largo de la prueba, y es acorde con la absorción de agua mostrada en la sección 4.3.

Se puede observar en la figura 5(b), la presencia formas características de carbonatos y verificado por EDS. Los carbonatos se forman a partir del $\mathrm{CO}_{2}$ de la atmósfera y de reacciones en la amorfa, le confiere excelentes propiedades mecánicas explicadas anteriormente en la prueba de Difracción de Rayos X.

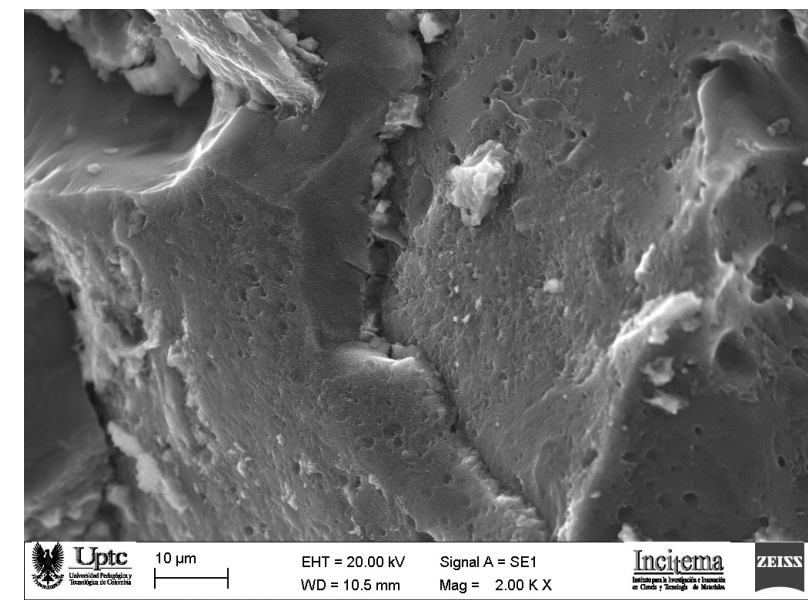

(a) a $10 \mu m$ y con $\mathrm{WD}=10.5 \mathrm{~mm}$

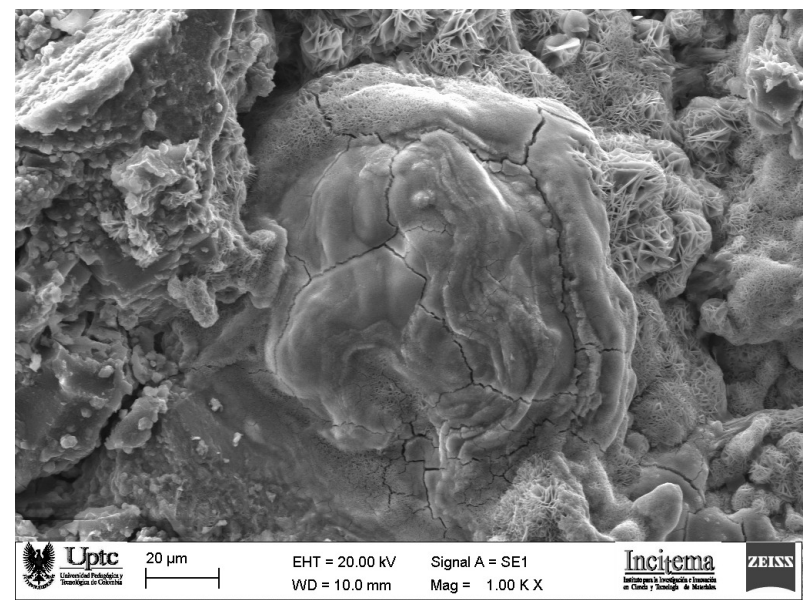

(b) a $20 \mu m$ y con $\mathrm{WD}=10.0 \mathrm{~mm}$

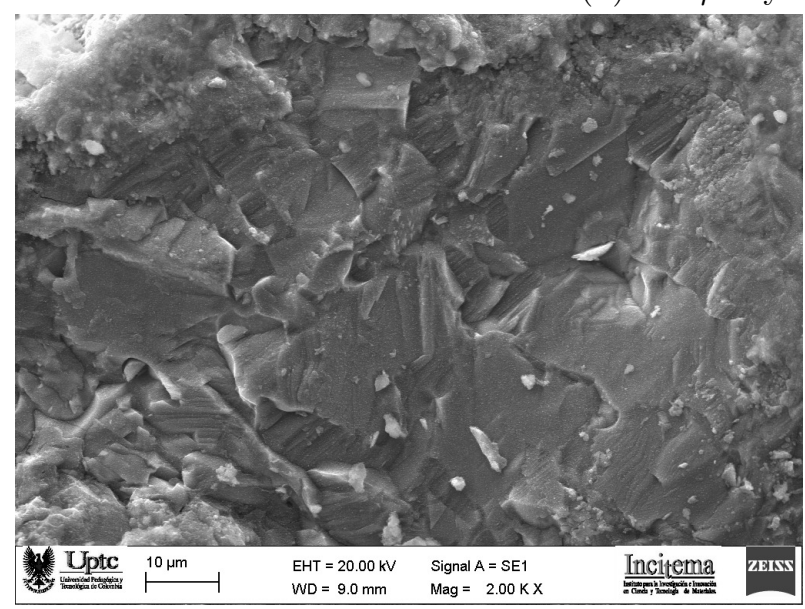

(c) a $20 \mu \mathrm{m}$ y con $\mathrm{WD}=9.0 \mathrm{~mm}$

Figura 5: Microscópio Electronico de Barrido de la muestra del Geopolímero EB70-CH10-SS15-HS5

En la Figura 5(c) se puede observar la formación de maclaje característico de las escorias, las zonas claras son partículas que parcialmente reaccionaron con el hidróxido.

\subsection{Discusión}

Las caracterizaciones físicas y químicas de las materias primas permitieron hacer diferentes observaciones sobre su calidad, entre las cuales se encuentra que la muestra que obtuvo mejor comportamiento mecánico (EB70-CH10-SS15-HS5), en la cual se formó Periclase 7.8\%, Calcita $15.0 \%$, Cuarzo $50.1 \%$, Cristobalita $9.6 \%$. Si se compara con los valores iniciales de Calcita $3.1 \%$, Illita $2.5 \%$, cuarzo $12 \%$, Cristobalita $18.9 \%$. Hubo una transformación de compuestos 
presentes en el momento de homogeneizar y solidificar la mezcla, esta transformación se hizo hacia compuestos característicos de los geopolímeros, es decir, se proporcionó un ambiente alcalino ideal para la disolución adecuada de partículas de Escoria Blanca y al mismo tiempo, el proceso de poli-condensación no se vio obstaculizado.

La carbonatación general de la estructura se vio incrementada en un $25,8 \%$, en esta clase de estructuras es un fenómeno generalmente natural que produce la neutralización (en términos de $\mathrm{pH}$ ), por cuanto, este aumento de la alcalinidad se debe al contacto de la pasta en solidificación con elementos acidos que se encuentran en el medio ambiente que lo rodea, en su mayoria, dióxido de carbono $\left(\mathrm{CO}_{2}\right)$ que se incorpora en la pasta y logra reaccionar.

De acuerdo con la figura 4, durante el proceso se formó Andradite $\left(\mathrm{Ca}_{3} \mathrm{Fe}_{2} \mathrm{Si}_{3} \mathrm{O}_{12}\right)$, compuesto característico de los geopolímeros en reacción y se asocia a carbonatos que reaccionaron con la illita, para la formación de andradita, dado el ambiente altamente alcalino de la solidificación.

Si se compararan los resultados obtenidos con la norma ICONTEC 4026, que establece los parámetros para el uso de concretos en mampostería. Se puede concluir que cumple totalmente con los parámetros requeridos de $11 M P a$ de resistencia a la compresión, que está bajo los rangos de absorción de agua, y se recomienda clasificarlo dentro del peso liviano es decir hasta $1680 \mathrm{~kg} / \mathrm{m}^{3}$, teniendo en cuenta que, por la naturaleza geopolimérica del material hasta el momento no existe una Norma Técnica Internacional que reglamente el uso de estos materiales, por considerarse relativamente nuevos.

\section{Conclusiones}

Los resultados indican la factibilidad del uso de Escoria blanca como precursor químico en la formación y estudio de estructuras geopoliméricas con buenas propiedades mecánicas, gracias a contenidos en la Escoria superiores al $20 \% \mathrm{SiO}_{2}, 40 \%$ de $\mathrm{CaO}$ y $5 \%$ de $\mathrm{Al}_{2} \mathrm{O}_{3}$, que se activan químicamente con hidróxido de sodio en un medio altamente alcalino para la formación de carbonatos de calcio, y otros compuestos químicos de mayor resistencia característicos de los material geopoliméricos, que cumple con la Norma Técnica Colombiana de resistencia a la compresión para ladrillos de mampostería estructural de viviendas, además, cumple con la norma ASTM C 69 y 89 de absorción de agua, con lo cual se proyecta buenas propiedades tribológicas.

El Silicato de Sodio y el chamote pueden ser considerados adiciones activas, que adquirieron la capacidad suficiente de reacción con el hidróxido de sodio en un ambiente alcalino, para aportar de manera efectiva en la resistencia a la compresión de las pastas geopoliméricas durante 28 días. .

La formación andradita y calcita indica la formación de estructuras propias de los geopolímero durante el trabajo, y que las proporciones son adecuadas para la síntesis de este geopolímeros. Aunque la resistencia a la compresión del geopolímero no es tan alta como otros sintetizados hasta el momento, se podría deber a la ausencia de Mullita en la estructura cristalina.

\section{Referencias}

[1] F. Pacheco; K. Zig-Zag, Ïntroduction to eco-efficient masonry bricks and blocks. ${ }^{\mathrm{Ec}} \mathrm{O}-$ Efficient Masonry Brick and Blocks, Design, Properties and Durability, Cap 1, pp 1-10, 2015.

[2] C. Leyva y D. Martinez, . Evaluación Técnico-Económica EmoS Aplicados A Los Procesos Convencionales De Producción En Una Empresa Ladrillera", Bogota: Universidad de la Salle, 2008.

[3] Deborah Olukemi Olanrewaju, "Lightweight Concrete Using Local Industrial By-product", Journal of Mechanics Engineering and Automation, vol. 4, no. 6, pp. 505-510, 2014.

[4] J. Pulido, J. Lizarazo-Marriaga, Comportamiento Mecánico de sistemas cementantes ternarios (Cemento Portland, Ceniza de Volante, Escoria de Alto Horno), LatinAmerican Journal of Metallurgy and Materials, vol. 36, no 2, pp 201-206, 2016

[5] O. Arioz, K. Kilinc, M. Tuncan, A. Tuncan, T. Kavas. "Physical, mechanical and micro-structural properties of F type fly-ash based geopolymeric bricks produced by pressure forming process". Adv Sci Technol, vol 69, pp 69-74, 2010. . 
[6] C. Chen, Q. Li, L. Shen and J. Zhai, "Feasibility of manufacturing geopolymer bricks using circulating fluidized bed combustion bottom ash", Environmental Technology, vol. 33, no. 11, pp. 1313-1321, 2012.

[7] T. Sheikh and M. Reza, "Production of Eco-Friendly Bricks from Copper Mine Tailings through Geopolymerization in India", International Journal of Trend in Scientific Research and Development, vol. -1, no. -5 , pp. 435-451, 2017.

[8] G. Mucsi, R. Szabó, Á. Rácz, F. Kristály and S. Kumar, Çombined Utilization Of Red Mud And Mechanically Activated Fly Ash In Geopolymers", Rudarsko-geološko-naftni zbornik, vol. 34, no. 1, pp. 27-36, 2019.

[9] T. Sékou, D. Siné, T. Lanciné and C. Bakaridjan, "Synthesis and Characterization of a Red Mud and Rice Husk Based Geopolymer for Engineering Applications", Macromolecular Symposia, vol. 373, no. 1, p. 1600090, 2017.

[10] T. Falayi, . ${ }^{\text {A }}$ comparison between fly ash- and basic oxygen furnace slag-modified gold mine tailings geopolymers", International Journal of Energy and Environmental Engineering, 2019.

[11] M. López, Y. Pineda and O. Gutiérrez, . Evaluation of Durability and Mechanical Properties of the Cement Mortar Added with Slag Blast Furnace", Procedia Materials Science, vol. 9, pp. 367-376, 2015.

[12] H. Peng, J. Yin and W. Song, "Mechanical and Hydraulic Behaviors of Eco-Friendly Pervious Concrete Incorporating Fly Ash and Blast Furnace Slag", Applied Sciences, vol. 8, no. 6, p. 859, 2018.

[13] P. Viera and C. Chicaiza, Üso De Residuos Cerámicos Como Sustituto Del Cemento Para Fabricación De Morteros", Ciencia, vol. 20, no. 1, p. 34, 2018.

[14] K. El-Naggar, S. Amin, S. El-Sherbiny and M. Abadir, "Preparation of geopolymer insulating bricks from waste raw materials", Construction and Building Materials, vol. 222, pp. 699-705, 2019.

[15] M. Zawrah, R. Gado, N. Feltin, S. Ducourtieux and L. Devoille, Recycling and utilization assessment of waste fired clay bricks (Grog) with granulated blast-furnace slag for geopolymer production", Process Safety and Environmental Protection, vol. 103, pp. 237-251, 2016.

[16] A. Özcan and M. Karakoç, Evaluation of sulfate and salt resistance of ferrochrome slag and blast furnace slag?based geopolymer concretes", Structural Concrete, vol. 20, no. 5, pp. 1607-1621, 2019.

[17] N. Abdel-Ghani, H. Elsayed and S. AbdelMoied, "Geopolymer synthesis by the alkali-activation of blastfurnace steel slag and its fire-resistance", HBRC Journal, vol. 14, no. 2, pp. 159-164, 2018.

[18] Norma ASTM C 39-15, Standard test method for compressive strength of cylindrical concrete specimens, vol. 04.02 American Society of Testing and Materials (ASTM), , Filadelfia (EE.UU.), 2015

[19] ASTM C1585-13, Standard Test Method for Measurement of Rate of Absorption of Water by Hydraulic-Cement Concretes, ASTM International, West Conshohocken, PA, 2013.

[20] J. Davitovits, M. Davidovics, Geopolymer: room-temperature ceramic matrix for composites, Ceramics Engineering Science Process, vol. 9 no 7, pp 835-842, 1998.

[21] J. Davitovits, Chemistry of Geopolymerics system, terminology, in: The Proceedings of Geopolymer 99, St. Quen Douglas c. Montgomery. Diseño y Análisis de Experimentos, Segunda Edición. Editorial Limusa. ISBN 968-18-6156-6. 71, France 1999, pp9-39.

[22] J. Davitovits, Global warming impact on the cement and aggregates industries, word Resource Review. vol. 6, n2, pp 263-278.

[23] J. Pulido, J. Lizarazo-Marriaga, W. Aperador, Çomportamiento mecánico de sistemas cementantes ternarios (cemento portland-ceniza volante-escoria de alto horno". Rev. LatinAm. Metal. Mat., vol. 36, n. 2, pp 201-216, 2016.

[24] A. Pap, K. Kordás, R. Peura and S. Leppävuori, "Simultaneous chemical silver and palladium deposition on porous silicon; FESEM, TEM, EDX and XRD investigation", Applied Surface Science, vol. 201, n. 1-4, pp. $56-60,2002$.

[25] E. Khankhaje et al., . ${ }^{\circ}$ blended cement and geopolymer concretes containing palm oil fuel ash", Materials \& Design, vol. 89, pp. 385-398, 2016.

[26] E. Furlani et al., "Synthesis and characterization of geopolymers containing blends of unprocessed steel slag and metakaolin: The role of slag particle size", Ceramics International, vol. 44, n. 5, pp. 5226-5232, 2018.

[27] O. Ogirigbo and L. Black, Ïnfluence of slag composition and temperature on the hydration and microstructure of slag blended cements", Construction and Building Materials, vol. 126, pp. 496-507, 2016.

[28] D. Ren, C. Yan, P. Duan, Z. Zhang, L. Li and Z. Yan, "Durability performances of wollastonite, tremolite and basalt fiber-reinforced metakaolin geopolymer composites under sulfate and chloride attack", Construction and Building Materials, vol. 134, pp. 56-66, 2017.

[29] P. Rozek, M. Król and W. Mozgawa, "Spectroscopic studies of fly ash-based geopolymers", Spectrochimica Acta Part A: Molecular and Biomolecular Spectroscopy, vol. 198, pp. 283-289, 2018.

[30] R. Antunes Boca Santa, C. Soares and H. Riella, "Geopolymers obtained from bottom ash as source of aluminosilicate cured at room temperature", Construction and Building Materials, vol. 157, pp. 459-466, 2017.

[31] T. Yang, H. Zhu and Z. Zhang, Ïnfluence of fly ash on the pore structure and shrinkage characteristics 
of metakaolin-based geopolymer pastes and mortars", Construction and Building Materials, vol. 153, pp. 284-293, 2017.

[32] A. Adamczyk, "The structural studies of aluminosilicate gels and thin films synthesized by the sol-gel method using different $\mathrm{Al}_{2} \mathrm{O}_{3}$ and $\mathrm{SiO}_{2}$ precursors", Materials Science-Poland, vol. 33, n. 4, pp. 732-741, 2015.

[33] V. Nikolić, M. Komljenović, Z. Baščarević, N. Marjanović, Z. Miladinović and R. Petrović, "The influence of fly ash characteristics and reaction conditions on strength and structure of geopolymers", Construction and Building Materials, vol. 94, pp. 361-370, 2015.

[34] S. Alonso and A. Palomo, Çalorimetric study of alkaline activation of calcium hydroxide-metakaolin solid mixtures", Cement and Concrete Research, vol. 31, n. 1, pp. 25-30, 2001.

[35] C. Yip, G. Lukey and J. van Deventer, "The coexistence of geopolymeric gel and calcium silicate hydrate at the early stage of alkaline activation", Cement and Concrete Research, vol. 35, n. 9, pp. 1688-1697, 2005.

[36] C. Yip, G. Lukey, J. Provis and J. van Deventer, . Effect of calcium silicate sources on geopolymerisation", Cement and Concrete Research, vol. 38, n. 4, pp. 554-564, 2008.

[37] C. Yip, J. Provis, G. Lukey and J. van Deventer, Çarbonate mineral addition to metakaolin-based geopolymers", Cement and Concrete Composites, vol. 30, n. 10, pp. 979-985, 2008.

[38] J. Qian and M. Song, "Study on Influence of Limestone Powder on the Fresh and Hardened Properties of Early Age Metakaolin Based Geopolymer", RILEM Bookseries, pp. 253-259, 2015.

[39] I. Perná and T. Hanzlíček, "The setting time of a clay-slag geopolymer matrix: the influence of blast-furnace-slag addition and the mixing method", Journal of Cleaner Production, vol. 112, pp. 1150-1155, 2016.

[40] T. Yang, Q. Wu, H. Zhu and Z. Zhang, "Geopolymer with improved thermal stability by incorporating high-magnesium nickel slag", Construction and Building Materials, vol. 155, pp. 475-484, 2017.

[41] J. Rogers, K. MacKenzie and W. Trompetter, "New phosphors synthesised by ion exchange of a metakaolin-based geopolymer", Applied Clay Science, vol. 157, pp. 1-7, 2018.

[42] S. Wattanasiriwech, F. Arif Nurgesang, D. Wattanasiriwech and P. Timakul, Çharacterisation and properties of geopolymer composite part 1: Role of mullite reinforcement", Ceramics International, vol. 43, n. 18, pp. 16055-16062, 2017.

[43] D. Montgomery, Diseño y análisis de experimentos, 2nd ed. [México]: Grupo Editorial Iberoamérica, 1991, pp. 71.
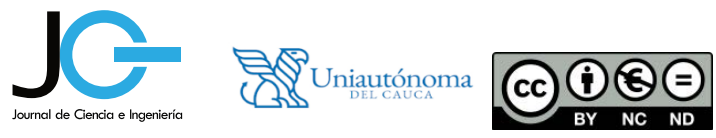